\title{
Scaling Law Describes the Spin-Glass Response in Theory, Experiments, and Simulations
}

\author{
Q. Zhai, ${ }^{1, *}$ I. Paga,,${ }^{2,3,}$ M. Baity-Jesi, ${ }^{4}$ E. Calore, ${ }^{5}$ A. Cruz, ${ }^{6,7}$ L. A. Fernandez, ${ }^{3,7}$ J. M. Gil-Narvion, ${ }^{7}$ I. Gonzalez-Adalid \\ Pemartin, ${ }^{3}$ A. Gordillo-Guerrero, ${ }^{8,9,7}$ D. Iñiguez, ${ }^{7,10}$ A. Maiorano, ${ }^{11,12,7}$ E. Marinari, ${ }^{13,12}$ V. Martin-Mayor, ${ }^{3,7}$ \\ J. Moreno-Gordo, ${ }^{7,6}$ A. Muñoz-Sudupe, ${ }^{3,7}$ D. Navarro, ${ }^{14}$ R. L. Orbach, ${ }^{1}$ G. Parisi,${ }^{15,12}$ S. Perez-Gaviro, ${ }^{16,7,6}$ \\ F. Ricci-Tersenghi, ${ }^{13,12}$ J. J. Ruiz-Lorenzo, ${ }^{17,9,7}$ S. F. Schifano, ${ }^{18}$ D. L. Schlagel, ${ }^{19}$ B. Seoane, ${ }^{3,7}$ A. Tarancon, ${ }^{6,7}$ \\ R. Tripiccione, ${ }^{5}$ and D. Yllanes $\oplus^{20,7, \dagger}$ \\ ${ }^{1}$ Texas Materials Institute, The University of Texas at Austin, Austin, Texas 78712, USA \\ ${ }^{2}$ Dipartimento di Fisica, Sapienza Università di Roma, INFN, Sezione di Roma I-00185, Italy \\ ${ }^{3}$ Departamento de Física Teórica, Universidad Complutense, 28040 Madrid, Spain \\ ${ }^{4}$ Eawag, Überlandstrasse 133, CH-8600 Dübendorf, Switzerland \\ ${ }^{5}$ Dipartimento di Fisica e Scienze della Terra, Università di Ferrara e INFN, Sezione di Ferrara, I-44122 Ferrara, Italy \\ ${ }^{6}$ Departamento de Física Teórica, Universidad de Zaragoza, 50009 Zaragoza, Spain \\ ${ }^{7}$ Instituto de Biocomputación y Física de Sistemas Complejos (BIFI), 50018 Zaragoza, Spain \\ ${ }^{8}$ Departamento de Ingeniería Eléctrica, Electrónica y Automática, Universidad de Extremadura, 10003 Cáceres, Spain \\ ${ }^{9}$ Instituto de Computación Científica Avanzada (ICCAEx), Universidad de Extremadura, 06006 Badajoz, Spain \\ ${ }^{10}$ Fundación ARAID, Diputación General de Aragón, Zaragoza, Spain \\ ${ }^{11}$ Dipartimento di Biotecnologie, Chimica e Farmacia, Università degli studi di Siena, 53100 Siena, Italy \\ ${ }^{12}$ INFN, Sezione di Roma 1, I-00185 Rome, Italy \\ ${ }^{13}$ Dipartimento di Fisica, Sapienza Università di Roma, and CNR-Nanotec, I-00185 Rome, Italy \\ ${ }^{14}$ Departamento de Ingeniería, Electrónica y Comunicaciones and I3A, Universidad de Zaragoza, 50018 Zaragoza, Spain \\ ${ }^{15}$ Dipartimento di Fisica, Sapienza Università di Roma, INFN, and CNR-Nanotec, I-00185 Rome, Italy \\ ${ }^{16}$ Escuela Universitaria Politécnica-La Almunia, 50100 La Almunia de Doña Godina, Zaragoza, Spain \\ ${ }^{17}$ Departamento de Física, Universidad de Extremadura, 06006 Badajoz, Spain \\ ${ }^{18}$ Dipartimento di Scienze Chimiche e Farmaceutiche, Università di Ferrara e INFN Sezione di Ferrara, I-44122 Ferrara, Italy \\ ${ }^{19}$ Division of Materials Science and Engineering, Ames Laboratory, Ames, Iowa 50011, USA \\ ${ }^{20}$ Chan Zuckerberg Biohub, San Francisco, California 94158, USA
}

(Received 7 July 2020; accepted 21 October 2020; published 30 November 2020)

\begin{abstract}
The correlation length $\xi$, a key quantity in glassy dynamics, can now be precisely measured for spin glasses both in experiments and in simulations. However, known analysis methods lead to discrepancies either for large external fields or close to the glass temperature. We solve this problem by introducing a scaling law that takes into account both the magnetic field and the time-dependent spin-glass correlation length. The scaling law is successfully tested against experimental measurements in a CuMn single crystal and against large-scale simulations on the Janus II dedicated computer.
\end{abstract}

DOI: 10.1103/PhysRevLett.125.237202

The dynamical arrest found upon cooling glass formers (spin glasses, fragile molecular glasses, polymers, colloids, etc.) to their glass temperature $T_{g}$ is a major open problem $[1,2]$. In the longstanding description [3], this slowing down is caused by the unbounded expansion of cooperative regions as $T_{g}$ is approached or as the system is left to age below $T_{g}$, which, in turn, leads to growing free-energy barriers. A quantitative description of this process is usually attempted in terms of a correlation length $\xi$. Unfortunately, in numerical simulations it is extremely difficult to measure the quantities that are easily accessible to experiments (and vice versa), which has led to seemingly irreconcilable approaches to the computation of the correlation length. On the one hand, theorists study correlation functions in an abstract replica space [4-14]. On the other hand, experimentalists measure the system's response to an applied external field (either an electric field for glass-forming liquids [15] or a magnetic field for spin glasses [16-20]). Reference [12] reconciled the two approaches by measuring the experimental response functions in a numerical simulation, but it was ultimately based on an approximate scaling law that breaks down for large fields or close to the glass temperature $T_{g}$. This is especially problematic, since temperatures $T \approx T_{g}$ are the most relevant for the study of glass formers ( $\xi$ is restricted to a very narrow window of variation if we move away from $T_{q}$ ).

Here, we are able to solve this dilemma in a framework that completely harmonizes experiments with theory. We conduct a parallel study of nonequilibrium spin-glass dynamics both in an experiment in a CuMn single crystal and in a large-scale simulation of the Ising-EdwardsAnderson (IEA) model carried out on the Janus II 
custom-built supercomputer [21]. We introduce a scaling law that describes the system's response over its entire natural range of variation.

To be specific, let us consider the zero-field-cooled protocol (see, e.g., [20]), where the spin glass is suddenly quenched from a temperature well above $T_{g}$ down to the working temperature $T_{m}<T_{g}$ and is then left to relax for a time $t_{w}$ [the growth of the correlation length $\xi\left(t_{w}\right)$ is unbounded for $T<T_{g}$, but very slow]. At time $t_{w}$, a magnetic field $H$ is applied and the growing magnetization $M\left(t, t_{w} ; H\right)$ is recorded for times $t+t_{w}$ (the $t_{w}$ dependence is included because spin glasses perennially age at $T<T_{g}$, slowly approaching equilibrium but never reaching it). The maximum of the relaxation function $d(M / H) / d \ln t$ defines a time $t_{H}^{\text {eff }}$ directly related to the height of the free-energy barriers that the system encounters. In a magnetic field, the Zeeman effect lowers these barriers by an amount proportional to $H^{2}$ and to the number of spins in a glassy cluster. Therefore, an Arrhenius law would predict a linear behavior of $\ln t_{H}^{\text {eff }}$ with $H^{2}$. Yet, see Fig. 1, departures from a straight line were observed for large values of $H^{2}$ in the very first experiment using this approach [16]. In fact, the Zeeman interpretation has been disputed [17,22] and identifying a linear behavior in $H^{2}$ becomes problematic close to $T_{g}$ [20].

In what follows, we shall derive a scaling law for the response to the magnetic field that is still valid for large

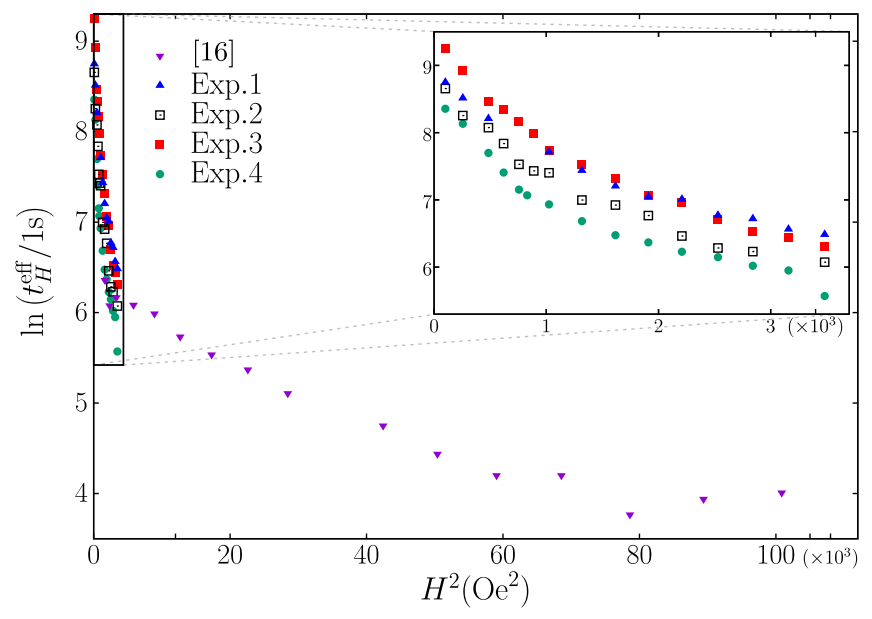

FIG. 1. Comparison of the classic experiment from Joh et al. [16] and present work. Both data sets are for $\mathrm{Cu}_{94} \mathrm{Mn}_{6}$. The data from Joh et al. are from a polycrystalline sample, while data from the present work come from a single crystal allowing for a much larger correlation length $\xi$ (see Table I for details). The figure shows the maximum of the relaxation function as a function of the squared magnetic field $H^{2}$. It is easy to estimate the slope at $H^{2}=0$ (from which $\xi$ is measured) for the data from Joh et al., which display a linear behavior for $H^{2} \lesssim 6 \times 10^{4} \mathrm{Oe}^{2}$. Instead, the large $\xi$ of the data from the present work not only causes a larger slope, but also a much larger curvature (see the enlarged region in the inset) which makes it challenging to extrapolate the slope to $H^{2}=0$. fields and close to $T_{g}$. As we stated above, the scaling law is tested against measurements in a single CuMn crystal and against massive numerical simulations carried out on Janus II. The single crystal is important because the growth of $\xi\left(t_{w}\right)$ is not limited like in a polycrystal with grain boundaries [23]. Specifically, we shall show that the $H$ dependence has the form

$$
\ln \frac{t_{H}^{\mathrm{eff}}}{t_{H \rightarrow 0^{+}}^{\mathrm{eff}}}=\frac{\hat{S}}{T} \xi^{D-(\theta / 2)} H^{2}+\xi^{-\theta / 2} \mathcal{G}\left(\xi^{D-(\theta / 2)} H^{2} ; T\right) .
$$

Here, $\xi$ stands for $\xi\left(t_{w}\right), \hat{S}$ is a constant, $D=3$ is the spatial dimension, and $\theta$ stands for the replicon exponent $\theta(\tilde{x})$ [4-6], where $\tilde{x}=\ell_{J}(T) / \xi\left(t_{w}\right)$ and $\ell_{J}(T)$ is the Josephson length $[13,20]$.

For small values of $x$ the scaling function behaves as $\mathcal{G}(x) \sim x^{2}\left(x=\xi^{D-(\theta / 2)} H^{2}\right)$. Hence, $\mathcal{G}$ is of order $H^{4}$ for small values of the magnetic field and, if $\xi$ is small (the typical case well below $T_{g}$ ), the contribution of $\mathcal{G}$ can be neglected for small $H$. In fact, most previous experiments and simulations only tested the $H^{2}$ term in Eq. (1). We find here, however, that for larger fields, or larger correlation lengths (which are found only close to $T_{g}$ ), $\mathcal{G}$ is the dominant contribution. Fortunately, Eq. (1) offers a unified framework that rationalizes the entire range of experiment and simulations.

Experimental and numerical descriptions.-Our experiments used a commercial dc SQUID to measure the magnetization of a $\mathrm{Cu}_{94} \mathrm{Mn}_{6}$ single crystal with $T_{g}=31.5 \mathrm{~K}$, grown at Ames Laboratory, U.S. DOE (see [20] for details). The sample was quenched from $40 \mathrm{~K}$ at $10 \mathrm{~K} / \mathrm{min}$ to the measuring temperature $T_{m}$ in zero magnetic field. After the temperature was stabilized, the system was aged for a waiting time $t_{w}$ before a magnetic field $H$ was turned on, and the magnetization $M_{\mathrm{ZFC}}\left(t, t_{w} ; T_{m}\right)$ was recorded as a function of time $t$. The temperatures were chosen as $28.5 \mathrm{~K}, 28.75 \mathrm{~K}$, and $29 \mathrm{~K}$, so $T_{m} \geq 0.9 T_{g}$. The magnetic fields ranged from 16 to 59 Oe. Table I shows the relevant experimental parameters, including the effective replicon exponent $\theta(\tilde{x})$.

In parallel with these experiments, we have simulated the Ising-Edwards-Anderson (IEA) model, with Hamiltonian $\mathcal{H}=-\sum_{\langle x, y\rangle} J_{x y} s_{x} s_{y}-H \sum_{x} s_{x}$, where $s_{x}= \pm 1$ is the

TABLE I. Main parameters for our four experiments, including the correlation length at time $t_{w}$ (in units of the average Mn-Mn spacing $a$ ) and the effective replicon exponent $\theta(\tilde{x})$, obtained from the interpolation in [20] of the results in [13].

\begin{tabular}{lcccc}
\hline \hline & $T_{m}(\mathrm{~K})$ & $t_{w}(\mathrm{~s})$ & $\xi\left(t_{w}\right) / a$ & $\theta(\tilde{x})$ \\
\hline Exp. 1 & 28.50 & 10000 & 320.36 & 0.337 \\
Exp. 2 & 28.75 & 10000 & 341.76 & 0.344 \\
Exp. 3 & 28.75 & 20000 & 359.18 & 0.342 \\
Exp. 4 & 29.00 & 10000 & 391.27 & 0.349 \\
\hline \hline
\end{tabular}


spin at site $\boldsymbol{x}$. We have used one sample of a cubic lattice with periodic boundary conditions, linear size $L=160$, and random couplings $J_{x y}= \pm 1$ [24]. In these natural units, and for $H=0$, the IEA model undergoes a spin-glass phase transition at the critical temperature $T_{g}=1.102(3)$ [26]. We simulated the nonequilibrium dynamics by means of a Metropolis algorithm. The natural time unit is the lattice sweep, which roughly corresponds to one picosecond of physical time. As for the magnetic field, Ref. [12] estimated from experimental $\mathrm{Fe}_{0.5} \mathrm{Mn}_{0.5} \mathrm{TiO}_{3}$ data [27] that $H=1$ in the IEA model corresponds to $5 \times 10^{4}$ Oe.

In order to mimic the experimental setup in the simulations, an initial random spin configuration is placed instantaneously at the working temperature $T_{m}$ and left to relax for a time $t_{w}$, with $H=0$. At time $t_{w}$, the external magnetic field is turned on and the magnetization $M\left(t, t_{w} ; H\right)$ and the correlation function $C\left(t, t_{w} ; H\right)=$ $\sum_{x} s_{x}\left(t_{w} ; H=0\right) s_{x}\left(t+t_{w} ; H\right) / 160^{3}$ are recorded.

Our experimental range (16 to 59 Oe) corresponds to $0.0003 \lesssim H \lesssim 0.0012$ in the IEA model, but the signal-tonoise ratio limited our simulations to $H \geq 0.005$. We employed two tricks to match these scales. On the one hand, we can use dimensional analysis [28] to relate $H$ and the reduced temperature $\hat{t}=\left(T_{g}-T\right) / T_{g}$ through

$$
\hat{t}_{\text {num }} \approx \hat{t}_{\exp }\left(\frac{H_{\text {num }}}{H_{\text {exp }}}\right)^{4 /[\nu(5-\eta)]},
$$

where $\nu=2.56(4)$ and $\eta=-0.390(4)$ are $H=0$ critical exponents [26], while the subscripts exp and num stand for experiment and simulation. Equation (2) suggests that we increase $\hat{t}_{\text {num }}$ to reach the experimental scale with our range or $H_{\text {num }}$, which results in $0.89 \lesssim T_{\text {num }} \lesssim 0.99$. Given our preexisting database of long simulations at $H=0$ [13], it has been convenient to work at temperatures $T_{m}=0.9$ and $T_{m}=1.0$ (or $\hat{t}=0.183$ and 0.093 ).

On the other hand, we have found that, when $H \rightarrow 0$, the correlation function $C\left(t, t_{w} ; H\right)$ approaches a constant value $C_{\text {peak }}$ at the maximum of the relaxation function [25], which suggests computing $t_{H}^{\text {eff }}$ in the simulations from the equation

$$
C\left(t_{H}^{\mathrm{eff}}, t_{w} ; H\right)=C_{\text {peak }} .
$$

See [29] for a similar choice in an equilibrium context. This is helpful because Eq. (3) can be solved at $H=0$ as well [in contrast with the magnetization, $C\left(t, t_{w} ; H\right)$ does not vanish at $H=0]$. The values of $C_{\text {peak }}$ are given in Table II.

The scaling law.-We work here on the same assumptions of Ref. [12], though we shall be able to improve on their findings.

In equilibrium and for large-enough correlation lengths, a scaling theory describes the magnetic response to an external field $H$ [30,31]. Our assumption will be (see also
TABLE II. Main parameters for our numerical simulations, including the replicon exponent $\theta(\tilde{x})$ and the value of $C_{\text {peak }}$ employed in Eq. (3).

\begin{tabular}{lccccc}
\hline \hline & $T_{m}$ & $t_{w}$ & $\xi\left(t_{w}, H=0\right)$ & $\theta(\tilde{x})$ & $C_{\text {peak }}$ \\
\hline Run 1 & 0.9 & $2^{22}$ & $8.294(7)$ & 0.455 & 0.530 \\
Run 2 & 0.9 & $2^{26.5}$ & $11.72(2)$ & 0.436 & 0.510 \\
Run 3 & 0.9 & $2^{31.25}$ & $16.63(5)$ & 0.415 & 0.490 \\
Run 4 & 1.0 & $2^{23.75}$ & $11.79(2)$ & 0.512 & 0.419 \\
Run 5 & 1.0 & $2^{27.625}$ & $16.56(5)$ & 0.498 & 0.400 \\
Run 6 & 1.0 & $2^{31.75}$ & $23.63(14)$ & 0.484 & 0.383 \\
\hline \hline
\end{tabular}

Refs. $[10,11])$ that this scaling theory holds as well in the nonequilibrium regime, at least for large $\xi\left(t_{w}\right)$ and small $H$ :

$$
\begin{aligned}
M\left(t, t_{w} ; H\right)= & {\left[\xi\left(t+t_{w}\right)\right]^{-(D / 2)-[\theta(\tilde{x}) / 4]} } \\
& \times \mathcal{F}\left(H\left[\xi\left(t+t_{w}\right)\right]^{-(D / 2)-[\theta(\tilde{x}) / 4]}, \frac{\xi\left(t+t_{w}\right)}{\xi\left(t_{w}\right)}\right),
\end{aligned}
$$

Because of (at least approximate) full-aging spinglass dynamics (see, e.g., [32]), Eq. (3) tells us that $\xi(t+$ $\left.t_{w}\right) / \xi\left(t_{w}\right)$ will be approximately constant close to the maximum of the relaxation rate (see Fig. 2), and we shall omit this dependence. Taylor expanding Eq. (4), and recalling that $\mathcal{F}(x)=-\mathcal{F}(-x)$, we find

$$
M\left(t, t_{w} ; H\right)=\chi_{1} H+\frac{\chi_{3}}{3 !} H^{3}+\frac{\chi_{5}}{5 !} H^{5}+\mathcal{O}\left(H^{7}\right),
$$

where [33]

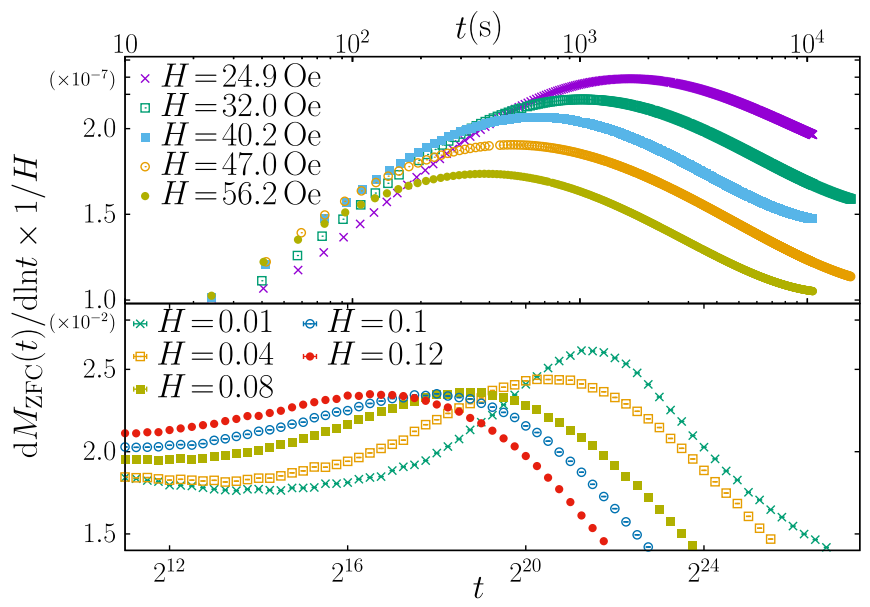

FIG. 2. A set of relaxation curves $S(t)=d(M / H) / d \ln t$ for CuMn at $T=29 \mathrm{~K}$ and $t_{w}=10^{4} \mathrm{~s}$ (top) and for the IsingEdwards-Anderson model at $T=0.9$ and $t_{w}=2^{22}$ lattice sweeps (bottom). The relation between IEA and physical units is discussed in the text. 


$$
\chi_{2 n-1} \propto b_{2 n}(T)\left[\xi\left(t_{w}\right)\right]^{(n-1) D-[n \theta(\tilde{x}) / 2]}
$$

$\left[b_{2 n}(T)\right.$ is a smooth function of $\left.T\right]$.

Our improvements over the results of [12] start from the observation that Eq. (6) predicts the paradoxical result $\chi_{1} \propto$ $\xi^{-\theta(\tilde{x}) / 2}$ (hence, $\chi_{1}$ would go to zero when $\xi \rightarrow \infty$ ). In fact, Eq. (6) neglects the contribution of the regular part of the free energy. A better description, then, is

$$
\chi_{1}=\frac{\hat{S}\left(C_{\mathrm{peak}}\right)}{T}+\frac{b_{2}(T)}{\xi^{\theta(\tilde{x}) / 2}},
$$

where $\hat{S}\left[C\left(t, t_{w}\right)\right]$ is the function appearing in the fluctuation-dissipation relations [34-37] [from now on, we use the shorthand $\hat{S}$ for $\left.\hat{S}\left(C_{\text {peak }}\right)\right]$.

Our next assumption will be that we can determine the excess free energy per spin in a field as it is done in equilibrium (by integrating $M$ with respect to $H$ )

$$
\Delta F=-\left[\frac{\chi_{1}}{2} H^{2}+\frac{\chi_{3}}{4 !} H^{4}+\frac{\chi_{5}}{6 !} H^{6}+\mathcal{O}\left(H^{8}\right)\right] .
$$

Equation (8), combined with Eqs. (6) and (7) leads directly to Eq. (1) when one makes a few additional hypothesis [38]: (i) according to an Arrhenius law, see [16,22,39], $t_{H}^{\mathrm{eff}} / t_{H=0}^{\mathrm{eff}}=\exp \left[N \Delta F /\left(k_{B} T\right)\right]$ where $N$ is the number of spins in a glassy domain, and (ii) $N \propto \xi^{D-\theta(\tilde{x}) / 2}$ [12].

The prefactor $\xi^{-\theta(\tilde{x}) / 2}$ for the $\mathcal{G}$ term in Eq. (1), not included in Ref. [12], will be crucial here because, unlike in [12], we shall test Eq. (1) in situations where the $\mathcal{G}$ term is the dominant contribution.

Experimental and numerical results.-We look at relaxation function curves exhibited in Fig. 2, from which the effective times $t_{H}^{\text {eff }}$ are obtained. Our results for $\ln t_{H}^{\text {eff }}$ (experiment) and $\ln t_{H}^{\mathrm{eff}} / t_{H=0}^{\mathrm{eff}}$ (simulations) are displayed in Fig. 3. The technical details about this analysis will appear elsewhere [25]. Both the experimental and the numerical data in Fig. 3 deviate very significantly from linear behavior, which suggests that the $\mathcal{G}$ term in Eq. (1) is, indeed, playing a dominant role.

Our next step is fitting the experimental data to

$$
\ln t_{H}^{\mathrm{eff}}=a_{0}+a_{2} H^{2}+a_{4} H^{4}+a_{6} H^{6}+\mathcal{O}\left(H^{8}\right) .
$$

Note that, in the experiments, $\ln t_{H}^{\text {eff }}$ needs to be extrapolated to $H=0$ (this is the meaning of the $a_{0}$ term). Our coefficients $a_{n}$ are listed in Table III. We extract $\xi$ from the $a_{2}$ term as explained in Ref. [20]. For the higher-order terms, Eqs. (1) and (5) predict $a_{n} \propto b_{2 n}(T) \xi^{[n D-(n+1) \theta(\tilde{x})] / 2}$. For instance, the $T=28.75 \mathrm{~K}$ data with $t_{w}^{(1)}=10 \mathrm{ks}$ and $t_{w}{ }^{(2)}=20 \mathrm{ks}$ allow a direct test of the scaling relation. Taking for $\theta(\tilde{x})$ the average value $\theta=0.343$ [25] we find

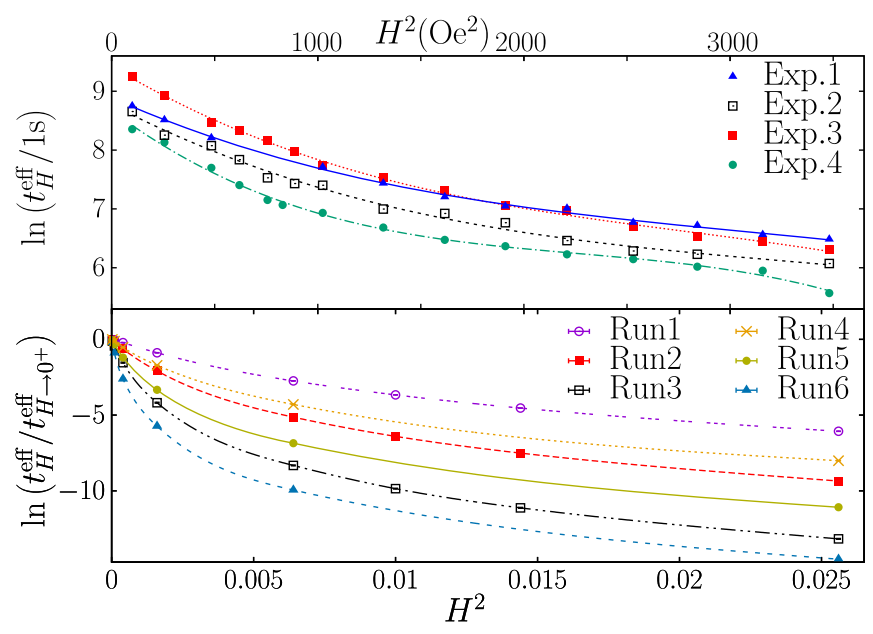

FIG. 3. Experimental and numerical $\ln t_{w}{ }^{\text {eff }}$ from the maximum of the response function in Fig. 2. Top: data from the experiments in Table I. Lines are fits to a polynomial in $H^{2}$, as in Eq. (9). The fit parameters are reported in Table III. Bottom: numerical data for the runs in Table II (the lines are just guides for the eye).

$\xi\left(t_{w}^{(2)}\right) / \xi\left(t_{w}^{(1)}\right)=\left[a_{2}\left(t_{w}^{(2)}\right) / a_{2}\left(t_{w}^{(1)}\right)\right]^{2 /(2 D-\theta)}=1.053$,

$\xi\left(t_{w}^{(2)}\right) / \xi\left(t_{w}^{(1)}\right)=\left[a_{4}\left(t_{w}^{(2)}\right) / a_{4}\left(t_{w}^{(1)}\right)\right]^{2 /(4 D-3 \theta)}=1.048$,

$\xi\left(t_{w}^{(2)}\right) / \xi\left(t_{w}^{(1)}\right)=\left[a_{6}\left(t_{w}^{(2)}\right) / a_{6}\left(t_{w}^{(1)}\right)\right]^{1 /(3 D-2 \theta)}=1.052$.

We can, therefore, gain access to the $\mathcal{G}$ term in Eq. (1) by subtracting $a_{0}+a_{2} H^{2}$ from the experimental value of $\ln t_{H}^{\mathrm{eff}}$.

As for the numerical data, polynomial fits analogous to Eq. (9) are possible, but result in wildly oscillating curves. The simplest explanation for this behavior is that our largest magnetic fields are beyond the radius of convergence of the Taylor expansion of Eq. (1). One can, however, compute $a_{2}$ by estimating the derivative of $\ln \left(t_{H}^{\mathrm{eff}} / t_{H \rightarrow 0^{+}}^{\mathrm{eff}}\right)$ numerically

TABLE III. Experimental data: coefficients $a_{n}$ of the polynomial fit of $\ln t_{w}^{\text {eff }}$, see Eq. (9), as a function of $T_{m}$ and $t_{w}$.

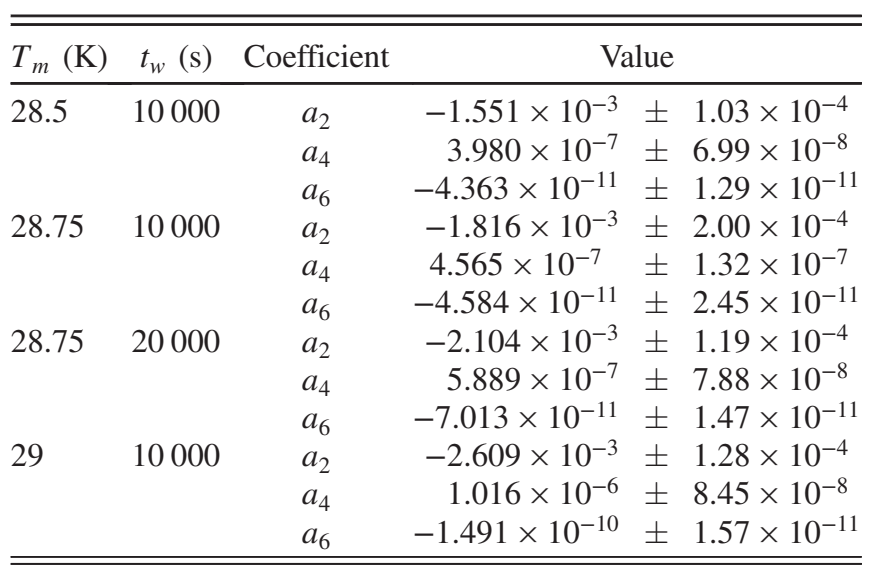




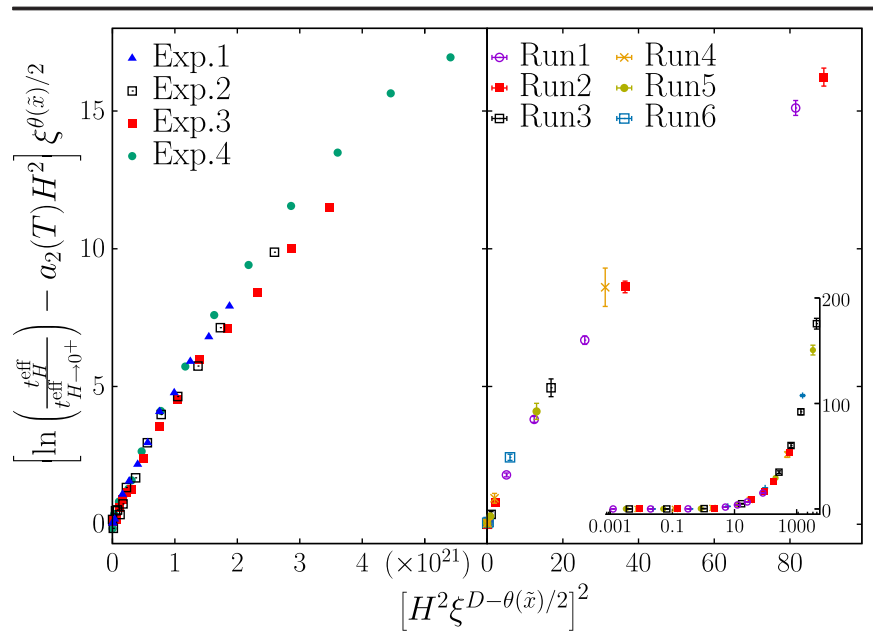

FIG. 4. The nonlinear part of the response time data: $\left[\ln \left(t_{H}^{\text {eff }} / t_{H \rightarrow 0^{+}}^{\text {eff }}\right)-a_{2}(T) H^{2}\right] \xi^{\theta(\tilde{x}) / 2}$ plotted against the scaling variable $\left[H^{2} \xi^{D-\theta(\tilde{x}) / 2}\right]^{2}$, see Eq. (1). Left: experimental data (see Table I). Right: numerical data (see Table II). The main panel, in linear scale, shows a closeup for small values of $\left[H^{2} \xi^{D-\theta(\tilde{x}) / 2}\right]^{2}$. The inset is in log scale in order to report all our numerical data. Note that experimental and numerical data are reported in different unit systems (see main text).

at $H^{2}=0$ [25]. Hence, we can access the $\mathcal{G}$ term in Eq. (1) with the same subtraction that we used for the experimental data.

Finally, Fig. 4 brings these analyses together to perform a strong test of Eq. (1) (assuming that the coefficients $b_{4}$ and $b_{6}$ are almost constant in the temperature range of interest). The agreement with the scaling prediction, manifested in a data collapse, is striking both for the experimental and the numerical data [40].

Conclusions. - The melding of experiment, theory, and simulations, as exhibited in Figs. 2-4, is a spectacular success of statistical mechanics. If the right questions are asked, a truly schematic model (namely the Ising-EdwardsAnderson model) turns out to behave, quantitatively, in the same way that CuMn does. The crucial ingredients to uncover this universal behavior have been high-quality simulations carried out on a custom-built computer, careful experiments capable of addressing the relevant regime of very large correlation lengths close to the glass temperature, and an extension to the nonequilibrium context of the classical equilibrium scaling theory. We are now able to model quantitatively-in a framework that encompasses both experiments and numerical simulations-responses, autocorrelation lengths, and energy barriers in threedimensional spin glasses. This will allow us to address more exotic phenomena such as rejuvenation (temperature chaos) and memory effects. Moreover, because spin glasses are influential in so many other fields (such as econophysics, biology, or optimization in computer science), our work shows that successful modeling of complex systems is feasible in finite dimensions.
We are grateful for helpful discussions with S. Swinnea about sample characterization. This work was partially supported by the U.S. Department of Energy, Office of Basic Energy Sciences, Division of Materials Science and Engineering, under Award No. DE-SC0013599, and Contract No. DE-AC02-07CH11358; by the Ministerio de Economía, Industria y Competitividad (MINECO, Spain), Agencia Estatal de Investigación (AEI, Spain), and Fondo Europeo de Desarrollo Regional (FEDER, EU) through Grants No. FIS2016-76359-P, No. PID2019103939RB-I00, No. PGC2018-094684-B-C21, and No. PGC2018-094684-B-C22; by the Junta de Extremadura (Spain) and Fondo Europeo de Desarrollo Regional (FEDER, EU) through Grants No. GRU18079 and No. IB15013. This project has also received funding from the European Research Council (ERC) under the European Union's Horizon 2020 research and innovation program (Grant No. 694925-LotglasSy). D. Y. was supported by the Chan Zuckerberg Biohub and I. G. A. P. was supported by the Ministerio de Ciencia, Innovación y Universidades (MCIU, Spain) through FPU Grant No. FPU18/02665. B.S. was supported by the Comunidad de Madrid and the Complutense University of Madrid (Spain) through the Atracción de Talento program (Ref. 2019-T1/TIC-12776).

*These authors contributed equally to this work. 'david.yllanes@czbiohub.org

[1] A. Cavagna, Phys. Rep. 476, 51 (2009).

[2] P. Charbonneau, J. Kurchan, G. Parisi, P. Urbani, and F. Zamponi, Nat. Commun. 5, 3725 (2014).

[3] G. Adam and J. H. Gibbs, J. Chem. Phys. 43, 139 (1965).

[4] E. Marinari, G. Parisi, J. Ruiz-Lorenzo, and F. Ritort, Phys. Rev. Lett. 76, 843 (1996).

[5] F. Belletti et al. (Janus Collaboration), Phys. Rev. Lett. 101, 157201 (2008).

[6] F. Belletti, A. Cruz, L. A. Fernandez, A. Gordillo-Guerrero, M. Guidetti, A. Maiorano, F. Mantovani, E. Marinari, V. MartínMayor, J. Monforte, A. Muñoz Sudupe, D. Navarro, G. Parisi, S. Perez-Gaviro, J. J. Ruiz-Lorenzo, S. F. Schifano, D. Sciretti, A. Tarancon, R. Tripiccione, and D. Yllanes (Janus Collaboration), J. Stat. Phys. 135, 1121 (2009).

[7] R. Alvarez Baños, A. Cruz, L. A. Fernandez, J. M. Gil-Narvion, A. Gordillo-Guerrero, M. Guidetti, A. Maiorano, F. Mantovani, E. Marinari, V. Martín-Mayor, J. Monforte-Garcia, A. Muñoz Sudupe, D. Navarro, G. Parisi, S. Perez-Gaviro, J. J. Ruiz-Lorenzo, S. F. Schifano, B. Seoane, A. Tarancon, R. Tripiccione, and D. Yllanes (Janus Collaboration), Phys. Rev. Lett. 105, 177202 (2010).

[8] M. Manssen and A. K. Hartmann, Phys. Rev. B 91, 174433 (2015).

[9] M. Manssen, A. K. Hartmann, and A. P. Young, Phys. Rev. B 91, 104430 (2015).

[10] L. A. Fernández and V. Martín-Mayor, Phys. Rev. B 91, 174202 (2015).

[11] M. Lulli, G. Parisi, and A. Pelissetto, Phys. Rev. E 93, 032126 (2016). 
[12] M. Baity-Jesi, E. Calore, A. Cruz, L. A. Fernandez, J. M. Gil-Narvion, A. Gordillo-Guerrero, D. Iñiguez, A. Maiorano, E. Marinari, V. Martin-Mayor, J. Monforte-Garcia, A. Muñoz Sudupe, D. Navarro, G. Parisi, S. Perez-Gaviro, F. Ricci-Tersenghi, J. J. Ruiz-Lorenzo, S. F. Schifano, B. Seoane, A. Tarancon, R. Tripiccione, and D. Yllanes (Janus Collaboration), Phys. Rev. Lett. 118, 157202 (2017).

[13] M. Baity-Jesi, E. Calore, A. Cruz, L. A. Fernandez, J. M. Gil-Narvion, A. Gordillo-Guerrero, D. Iñiguez, A. Maiorano, E. Marinari, V. Martin-Mayor, J. Moreno-Gordo, A. Muñoz Sudupe, D. Navarro, G. Parisi, S. Perez-Gaviro, F. Ricci-Tersenghi, J. J. Ruiz-Lorenzo, S.F. Schifano, B. Seoane, A. Tarancon, R. Tripiccione, and D. Yllanes (Janus Collaboration), Phys. Rev. Lett. 120, 267203 (2018).

[14] L. A. Fernandez, E. Marinari, V. Martin-Mayor, I. Paga, and J. J. Ruiz-Lorenzo, Phys. Rev. B 100, 184412 (2019).

[15] S. Albert, T. Bauer, M. Michl, G. Biroli, J.-P. Bouchaud, A. Loidl, P. Lunkenheimer, R. Tourbot, C. Wiertel-Gasquet, and F. Ladieu, Science 352, 1308 (2016).

[16] Y. G. Joh, R. Orbach, G. G. Wood, J. Hammann, and E. Vincent, Phys. Rev. Lett. 82, 438 (1999).

[17] F. Bert, V. Dupuis, E. Vincent, J. Hammann, and J.-P. Bouchaud, Phys. Rev. Lett. 92, 167203 (2004).

[18] S. Guchhait and R. Orbach, Phys. Rev. Lett. 112, 126401 (2014).

[19] S. Guchhait and R. L. Orbach, Phys. Rev. Lett. 118, 157203 (2017).

[20] Q. Zhai, V. Martin-Mayor, D. L. Schlagel, G. G. Kenning, and R. L. Orbach, Phys. Rev. B 100, 094202 (2019).

[21] M. Baity-Jesi, R. A. Baños, A. Cruz, L. A. Fernandez, J. M. Gil-Narvion, A. Gordillo-Guerrero, D. Iniguez, A. Maiorano, F. Mantovani, E. Marinari, V. Martín-Mayor, J. Monforte-Garcia, A. Muñoz Sudupe, D. Navarro, G. Parisi, S. Perez-Gaviro, M. Pivanti, F. Ricci-Tersenghi, J. J. Ruiz-Lorenzo, S. F. Schifano, B. Seoane, A. Tarancon, R. Tripiccione, and D. Yllanes (Janus Collaboration), Comput. Phys. Commun. 185, 550 (2014).

[22] E. Vincent, J. P. Bouchaud, D. S. Dean, and J. Hammann, Phys. Rev. B 52, 1050 (1995).

[23] G. G. Kenning, D. M. Tennant, C. M. Rost, F. G. da Silva, B. J. Walters, Q. Zhai, D. C. Harrison, E. D. Dahlberg, and R. L. Orbach, Phys. Rev. B 98, 104436 (2018).
[24] In a $L=160$ system, $\xi\left(t_{w}\right)$ and $M\left(t, t_{w} ; H\right)$ display little sample dependence, see [25]. We have, however, run 512 independent thermal histories for our sample (the benefits of simulating many independent thermal histories are discussed in Ref. [13]).

[25] Q. Zhai et al. (to be published).

[26] M. Baity-Jesi et al. (Janus Collaboration), Phys. Rev. B 88, 224416 (2013).

[27] H. Aruga Katori and A. Ito, J. Phys. Soc. Jpn. 63, 3122 (1994).

[28] D. S. Fisher and H. Sompolinsky, Phys. Rev. Lett. 54, 1063 (1985).

[29] I. G.-A. Pemartín, V. Martin-Mayor, G. Parisi, and J. J. Ruiz-Lorenzo, J. Phys. A 52, 134002 (2019).

[30] G. Parisi, Statistical Field Theory (Addison-Wesley, Reading, MA, 1988).

[31] D. J. Amit and V. Martín-Mayor, Field Theory, the Renormalization Group and Critical Phenomena, 3rd ed. (World Scientific, Singapore, 2005).

[32] G. F. Rodriguez, G. G. Kenning, and R. Orbach, Phys. Rev. Lett. 91, 037203 (2003).

[33] In order to not overburden the notation we have omitted the $t$ and $t_{w}$ arguments in the rhs susceptibilities in Eq. (5).

[34] L. F. Cugliandolo and J. Kurchan, Phys. Rev. Lett. 71, 173 (1993).

[35] E. Marinari, G. Parisi, F. Ricci-Tersenghi, and J. J. Ruiz-Lorenzo, J. Phys. A 31, 2611 (1998).

[36] S. Franz, M. Mézard, G. Parisi, and L. Peliti, Phys. Rev. Lett. 81, 1758 (1998).

[37] M. Baity-Jesi et al., Proc. Natl. Acad. Sci. U.S.A. 114, 1838 (2017).

[38] For the sake of simplicity, we have neglected the correction of order $\xi^{-\theta / 2}$ in the $H^{2}$ term in Eq. (1).

[39] C. Djurberg, J. Mattsson, and P. Nordblad, Europhys. Lett. 29, 163 (1995).

[40] Note that the scaling form of Eq. (1) is necessary to obtain a good collapse. To support this, we show in the Supplemental Material [41] that the collapse of Fig. 4 deteriorates if one assumes different scaling behaviors.

[41] See Supplemental Material at http://link.aps.org/ supplemental/10.1103/PhysRevLett.125.237202 for a comparison with different scaling laws. 